\title{
MicroRNA-141 inhibits tumor growth and minimizes therapy resistance in colorectal cancer
}

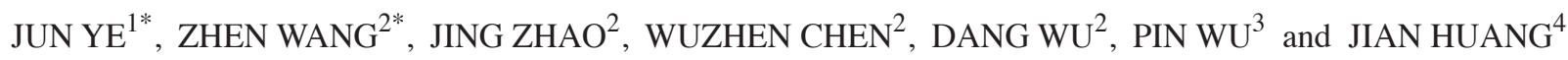 \\ ${ }^{1}$ Department of Gastroenterology; ${ }^{2}$ Cancer Institute (Key Laboratory of Cancer Prevention and Intervention, \\ China National Ministry of Education, Key Laboratory of Molecular Biology in Medical Sciences, Zhejiang); \\ Departments of ${ }^{3}$ Thoracic Surgury and ${ }^{4}$ Oncology, The Second Affiliated Hospital, \\ Zhejiang University School of Medicine, Hangzhou, Zhejiang 310009, P.R. China
}

Received November 2, 2015; Accepted October 28, 2016

DOI: $10.3892 / \mathrm{mmr} .2017 .6135$

\begin{abstract}
Colorectal cancer (CRC) is one of most common cancers and causes of cancer-associated mortality worldwide, due to its recurrence, metastasis and therapy resistance. Cancer stem cells (CSC) have been demonstrated to be vital for tumor initiation and recurrence. microRNAs may act as an oncogenes or tumor suppressors in numerous cancers. The present study demonstrated that microRNA-141 (miR-141) was downregulated in CSC compared with differentiated cancer cells, and in tumor compared with healthy tissue. miR-141 may inhibit CRC cell proliferation and the maintenance of CSC stemness, thereby enhancing drug susceptibility. In addition, the present study identified cyclin D2 as a novel target gene of miR-141. In conclusion, the antitumor role of miR-141 and its target cyclin D2 may suggest the development of miR-141 as a potential therapeutic agent.
\end{abstract}

\section{Introduction}

Colorectal cancer (CRC) is the third most prevalent cancer and the third most common cause of cancer-associated mortality in males and females worldwide (1). Despite rapid advances in diagnosis and treatment methods, including surgery and chemotherapy, prognosis remains poor. Certain patients suffer drug resistance, which is partially due to the presence of cancer stem cells (CSCs) (2).

Correspondence to: Professor Jian Huang, Department of Oncology, The Second Affiliated Hospital, Zhejiang University School of Medicine, 88 Jiefang Road, Hangzhou, Zhejiang 310009, P.R. China

E-mail: drhuangjian@zju.edu.cn

${ }^{*}$ Contributed equally

Key words: microRNA-141, colorectal cancer, cancer stem cell, cyclin D2, drug resistance
MicroRNAs (miRNAs) are a class of small, evolutionarily conserved, noncoding RNAs. miRNAs regulate various target genes by binding to the 3 ' untranslated region (3'UTR) of target mRNA, resulting in translational repression at the posttranscriptional level, via mRNA degradation, or through altering gene expression by interactions with gene promoters (3). miRNAs are important in biological and cellular processes, including proliferation, apoptosis, metabolism and differentiation $(4,5)$. Aberrant expression of miRNAs has been observed in numerous human diseases, including cancer, metabolic disease, autoimmune disease, cardiovascular disease and neurological disorders. Currently, it is widely accepted that miRNAs may act as oncogenes or suppressor genes during tumor development. For example, miR-27b has been demonstrated to inhibit CRC progression and angiogenesis via targeting vascular endothelial growth factor C, and miR-130b promotes liver CSC growth and self-renewal through targeting tumor protein P53 inducible nuclear protein 1 (6).

Considering that CSCs may be critical for tumor development and drug resistance, the present study aimed to investigate molecular differences between CSCs and differentiated cancer cells, including in miRNA expression. Numerous surface markers have been identified for CSC sorting, including cluster of differentiation CD133, CD24, CD44 and CD166 (7,8), of which CD133 is the most widely used. CD133 is a five-transmembrane glycoprotein with a molecular weight of $120 \mathrm{kDa}$. It has been demonstrated to identify CSCs in various human tumors, including prostate cancer, pancreatic cancer, leukemia, brain tumor, hepatocellular carcinoma, breast cancer and colon cancer (9-15).

Our previous study have established the Y cell line, which is enriched in $\mathrm{CD}_{133^{+}}$cells and exhibits distinct stemness characteristics, including sphere formation and the capacity for self-renewal (16). Using miRNA microarrays, the present study revealed that miR-141 expression is decreased in CSC-like Y cells and in CRC tissues. miR-141 is located on chromosome 12 and belongs to the same cluster as the miR-200 family. miR-141 has been observed to be downregulated in gastric cancer, renal cell carcinoma and breast cancer (17). However, the functions and potential targets of miR-141 in CRC cells remain to be fully elucidated. The present study demonstrated that miR-141 inhibited CRC cell proliferation via targeting cyclin D2, which 
is involved in cell cycle regulation, and inhibited the maintenance of CSC stemness, thereby enhancing drug susceptibility. Targeting miR-141 may therefore be a potential strategy for the treatment of CRC.

\section{Materials and methods}

Clinical colorectal cancer tissue sample collection and analysis. Tissue samples $(\mathrm{n}=8)$ were collected between January 2008 and December 2010 at the Second Affiliated Hospital, Zhejiang University School of Medicine (Hangzhou, China) and confirmed to be colorectal cancer pathologically. All participants provided written consent of their information to be stored in the hospital database and for samples to be used for research. This research was approved by the Institutional Review Boards of the Second Affiliated Hospital, Zhejiang University School of Medicine.

Cell lines. The Y human colorectal cancer cell line was established in our laboratory, as described previously (2). The HT29 and SW620 human colorectal adenocarcinoma cell line was obtained from the Cell Bank of the China Academy of Medical Sciences (Beijing, China). Y and HT29 cells were cultured in RPMI-1640 medium (Gibco; Thermo Fisher Scientific, Inc., Waltham, MA, USA) supplemented with $10 \%$ fetal bovine serum (FBS; Gibco; Thermo Fisher Scientific, Inc.). SW620 cells were cultured in Leibovitz L15 medium (Gibco; Thermo Fisher Scientific, Inc.) supplemented with FBS (Gibco; Thermo Fisher Scientific, Inc.). All cells were maintained at $37^{\circ} \mathrm{C}$ in a humidified $5 \% \mathrm{CO}_{2}$ atmosphere.

Flow cytometry. CRC cells $\left(<2 \times 10^{7}\right)$ were labeled with an anti-human monoclonal CD133 antibody conjugated to R-phycoerythrin (R-PE; cat. no. 130-098-826; $10 \mu 1$ per $10^{7}$ cells; Miltenyi Biotec $\mathrm{GmbH}$, Bergisch Gladbach, Germany) in PBS containing $2 \% \mathrm{FBS}$ for $10 \mathrm{~min}$ in the dark at $4^{\circ} \mathrm{C}$. A blank control and mouse IgG1-R-PE isotype control (cat. no. 130-098-845; $10 \mu \mathrm{l}$ per $10^{7}$ cells; Miltenyi Biotec $\mathrm{GmbH}$ ) served as controls . $\mathrm{CD}_{133^{+}}$cells were identified and sorted using a FACSCanto II flow cytometer (BD Biosciences, San Jose, CA, USA).

miRNA expression microarray analysis. Total RNA was isolated from $\mathrm{CD} 133^{+}$and CD133- CRC cells using TRIzol ${ }^{\circledR}$ reagent (Invitrogen; Thermo Fisher Scientific, Inc.) according to the manufacturer's protocol. The quantity and the quality of RNA were evaluated using a Nanodrop spectrophotometer (Thermo Fisher Scientific, Inc.). The miRNA expression profile of $\mathrm{CD}_{133^{+}}$and $\mathrm{CD} 133^{-} \mathrm{CRC}$ cells was assessed using an Affymetrix miRNA array (Affymetrix GeneChip miRNA 2.0 array; Affymetrix, Inc., Santa Clara, CA, USA).

Reverse transcription-quantitative polymerase chain reaction (RT-qPCR) analysis. Total RNA was isolated from cell lines and tissues using TRIzol reagent according to the manufacturer's protocol. The quantity and the quality of RNA were evaluated using a Nanodrop spectrophotometer. The RNA was reverse-transcribed to cDNA using Moloney Murine Leukemia Virus Reverse Transcriptase (Promega Corporation, Madison, WI, USA). The expression of miR-141, sex determining region Y-box 2 (Sox2), octamer-binding transcription factor 4 (Oct4), nanog homeobox (Nanog) and B cell-specific Moloney murine leukemia virus integration site 1 (Bmil) was measured using the SYBR ${ }^{\circledR}$-Green PCR Master mix (Applied Biosystems; Thermo Fisher Scientific, Inc.) and the StepOnePlus Real-Time PCR system (Applied Biosystems; Thermo Fisher Scientific, Inc.). All samples were run in triplicate. The miR-141 expression levels were normalized to those of U6, which served as an internal control. The Sox2, Oct4, Nanog and Bmil expression levels were normalized to those of GAPDH, which served as an internal control. The relative expression of target genes were calculated using the $2^{-\Delta \Delta C q}$ method (18). The primer sequences used were as follows: SOX2, F 5'-AACCCCAAGATGCAC AACTC-3', R 5'-CGGGGCCGGTATTTATAATC-3'; Oct4, F 5'-TTCTCAGGGGGACCAGTGTC-3'; R 5'-CCCATTCCT AGAAGGGCAGG-3'; Nanog, F 5'-CCAGTGACTTGGAGG CTGC-3', R 5'-AAGGATTCAGCCAGTGTCC-3'; Bmi1, F 5'-TGACAAATGCTGGAGAACTG-3', R 5'-AAGATTGGT GGTTACCGCT-3'; GADPH, F 5'-ACAGTCAGCCGCATC TTCTT-3'; R 5'-TGGAAGATGGTGATGGGATT-3'. Results were expressed as relative quantitation.

Proliferation assay. CRC cells were transfected with the negative control mimic (5'-UUCUCCGAACGUGUCACG UTT-3'), an miR-141 mimic (5'-UAACACUGUCUGGUA AAGAUGG-3') or an anti-miR-141 inhibitor (5'-CCA UCUUUACCAGACAGUGUUA-3'), all obtained from Shanghai GenePharma Co., Ltd. (Shanghai, China) using Lipofectamine $^{\circledR} 2000$ (Invitrogen; Thermo Fisher Scientific, Inc.) and incubated for $12 \mathrm{~h}$. Cells were subsequently seeded at a density of $3 \times 10^{3}$ cells per well in $0.2 \mathrm{ml}$ RPMI 1640 medium containing 10\% FBS in a 96-well plate. MTS reagent $(20 \mu \mathrm{l}$; Promega Corporation) was added to each well and the cells were incubated at $37^{\circ} \mathrm{C}$ for $4 \mathrm{~h}$. The absorbance values were measured at a wavelength of $490 \mathrm{~nm}$ on a microplate reader (Bio-Rad Laboratories, Inc., Hercules, CA, USA) and assessed every day for 3 consecutive days.

Cell viability assay. CRC cells were transfected as described above. After a further incubation of $24 \mathrm{~h}$, cells were reseeded in 96-well plates and treated with various concentrations of 5-fluorouracil (5-FU; Nantong Jinghua Pharmaceutical Co., Ltd., Nantong, China) or oxaliplatin (Sanofi S.A., Gentilly, France) for $48 \mathrm{~h}$. A proliferation assay was subsequently performed as described above, to determine the individual inhibitory concentration 50 values (50\% cell growth inhibitory concentrations).

Cell cycle analysis. Cells were trypsinized and fixed in $70 \%$ ethanol. The DNA content was assessed by analyzing incorporated propidium iodide (PI) $\left(10 \mu \mathrm{l} \mathrm{PI}\right.$ in $1.5 \mathrm{ml} 1 \times 10^{6}$ cells; Multi Sciences (Lianke) Biotech Co., Ltd., Hangzhou, China) using a FACScanto II. The cell populations in G0/G1, $\mathrm{S}$ and G2/M phases were determined using ModFit LT software v2.0 (Verity Software House, Inc., Topsham, ME, USA).

Western blotting. Total protein was extracted from cells lysed with the M-PER Mammalian Protein Extraction Reagent (Thermo Fisher Scientific, Inc.) supplemented with a protease inhibitor cocktail (Sigma-Aldrich; Merck Millipore, Darmstadt, Germany). Protein extracts (50 $\mu 1$ extract from 
A
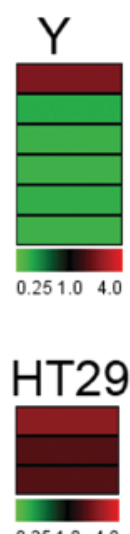

hsa-miR-373*

hsa-miR-29b hsa-miR-638 hsa-miR-19a hsa-miR-101 hsa-miR-141

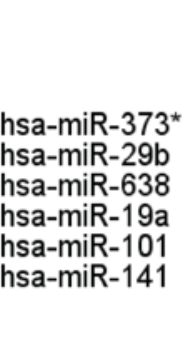

B

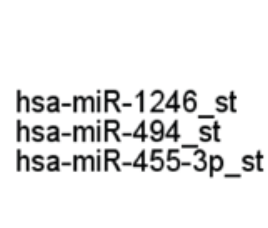

Figure 1. miR-141 expression levels in CSCs and CRC tumor tissue. (A) Differentially expressed miRNAs in CD133+ (a marker of CSCs) and CD133- cells. Red

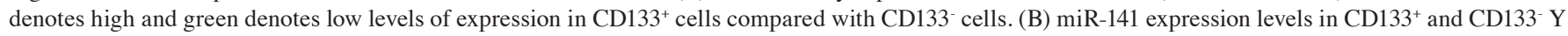
cells were verified using RT-qPCR. "P<0.05 vs. CD133'. (C) miR-141 expression levels in fresh CRC tissues and adjacent healthy tissues from eight patients, as assessed by RT-qPCR. The y-axis indicates the fold change. ${ }^{*} \mathrm{P}<0.05$ vs. paired sample. miR, microRNA; CSCs, cancer stem cells; CRC, colorectal cancer; $\mathrm{CD}$, cluster of differentiation; RT-qPCR, reverse transcription-quantitative polymerase chain reaction.

$1 \times 10^{6}$ cells) were separated by SDS-PAGE and transferred to a PVDF membrane. Following blocking with $5 \%$ non-fat milk in TBS containing Tween-20 (TBST) for $60 \mathrm{~min}$, the membrane was incubated with anti-human-cyclin D2 (cat. no. 3741S; 1:2,000; Cell Signaling Technology, Inc., Danvers, MA, USA) or anti-human GAPDH (cat no. KC-5G5; 1:2,000; Shanghai Kang-Chen Biotech, Shanghai, China). Primary antibodies were diluted in $5 \%$ bovine serum albumin (Amresco, LLC, Solon, OH, USA) in TBST and incubated overnight at $4^{\circ} \mathrm{C}$. Membranes were incubated in secondary antibody [anti-rabbit horseradish peroxidase conjugated; cat. no. 70-GAR007; 1:5,000; Multi Sciences (Lianke) Biotech Co., Ltd.) for $1 \mathrm{~h}$ at room temperature. ECL chromogenic substrate [cat no. 70-P1421; Multi Sciences (Lianke) Biotech Co., Ltd.] was used to visualize the proteins.

Statistical analysis. Data are presented as the mean \pm standard error using SPSS 17.0 (SPSS, Inc., Chicago, IL, USA). The RT-qPCR results from paired clinical samples were analyzed using a two-tailed paired Student's $t$-test and the remaining data were analyzed using a two-tailed unpaired Student's t-test or analysis of variance. $\mathrm{P}<0.05$ was considered to indicate a statistically significant difference.

\section{Results}

miR-141 expression levels decrease in colorectal CSCs. CSCs are involved in tumor initiation, recurrence, metastasis and chemotherapy resistance $(7,19)$. The present study assessed miRNA expression profiles of $\mathrm{CD} 133^{+}$and $\mathrm{CD}^{-} 33^{-}$cells to identify differentially expressed miRNAs potentially involved in tumor progression. Microarray analysis identified four miRNAs that were upregulated (miR-373, miR-1246-star, miR-494-star and miR-455-3p-star) and 5 that were downregulated (miR-29b, miR-638, miR-19a, miR-101 and miR-141) in $\mathrm{CD}_{133^{+}}$cells compared with CD133- cells (Fig. 1A). Of these, miR-141 underwent the greatest decrease. In addition, the importance of the miR-200 family in cancer development has been demonstrated, therefore miR-141 was selected for further investigation. RT-qPCR confirmed the microarray results, demonstrating a 2.33-fold decrease in miR-141 expression levels in $\mathrm{CD}_{133^{+}}$cells compared with $\mathrm{CD} 133^{-}$cells sorted from the $Y$ cell line (Fig. 1B).

miR-141 expression levels were analyzed in CRC tissue samples. In the limited number of fresh tissue samples available $(n=8)$, miR-141 expression was downregulated in the majority of CRC samples compared with adjacent healthy tissues (Fig. 1C). Thus, downregulation of miR-141 may be important during CRC development.

miR-141 inhibits tumor cell proliferation and increases chemotherapy sensitivity. CRC cells were transfected with a negative control mimic, an miR-141 mimic or an anti-miR-141 inhibitor to investigate the biological functions of miR-141. RT-qPCR confirmed the transfection efficiency. Overexpression of miR-141 suppressed cell proliferation, whereas inhibiting miR-141 via an anti-miR-141 promoted cell proliferation (Fig. 2A).

The effect of miR-141 on drug resistance was examined in vitro. 5-FU or oxaliplatin as typical antitumor drugs were added into the culture medium of SW620 and Y cells. Transfection with anti-miR-141 enhanced drug resistance (Fig. 2B). Following treatment of SW620 and Y cells with various concentrations $(2,5$ or $10 \mathrm{ug} / \mathrm{ml})$ of 5 -FU (Fig. 2C) or oxaliplatin (Fig. 2D), cell viability was markedly reduced in the miR-141 mimic-transfected, compared with the negative control, group. Transfection with anti-miR-141 demonstrated the opposite effect. These results indicated that miR-141 suppresses CRC proliferation and enhances drug sensitivity.

miR-141 promotes stem cell differentiation in CRC. Sox2, Oct4, Nanog and Bmil are stem cell-associated genes, which are involved in self-renewal and the maintenance of stemness in stem cells (20). Following transfection of SW620 cells with 

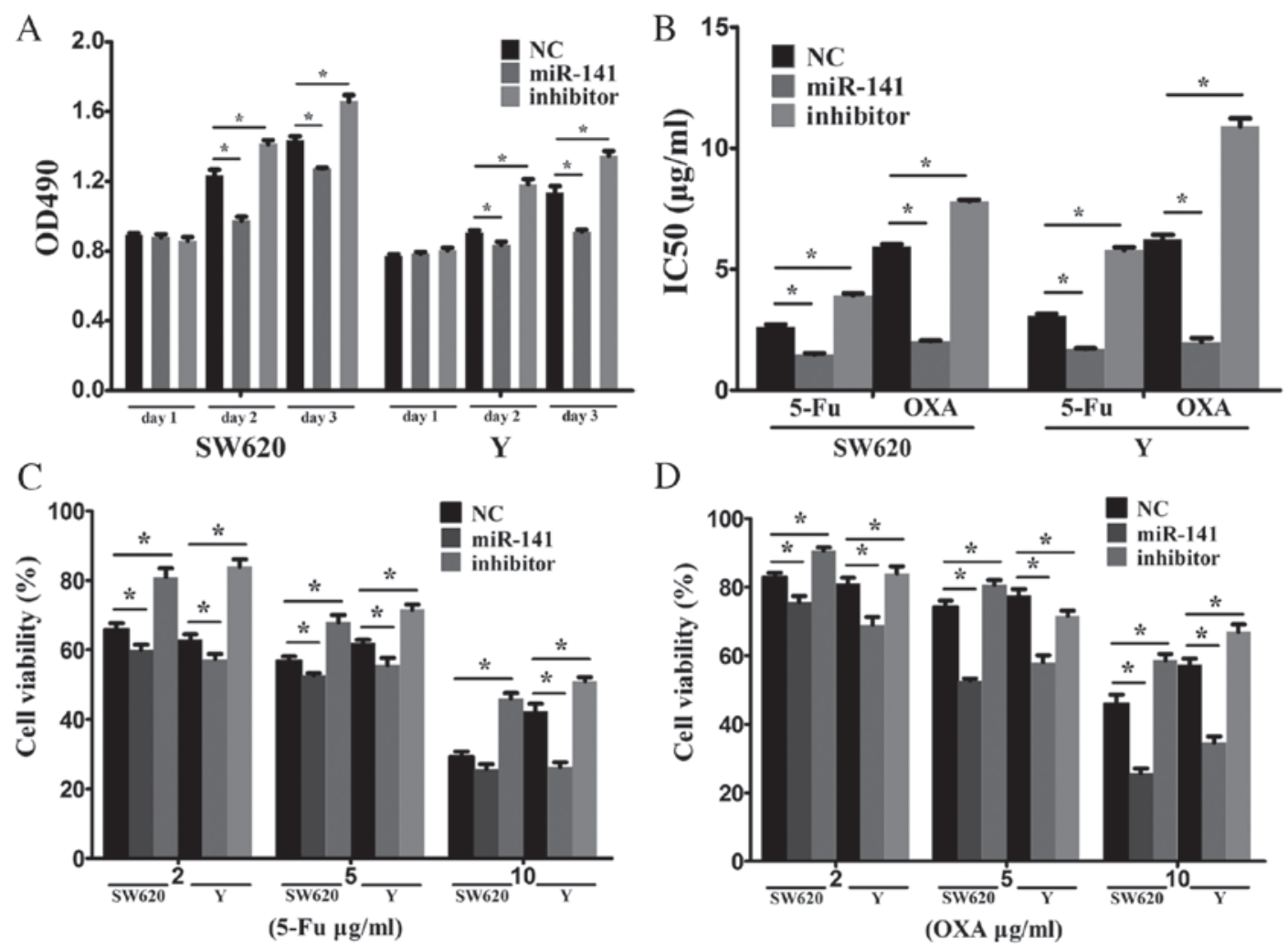

D

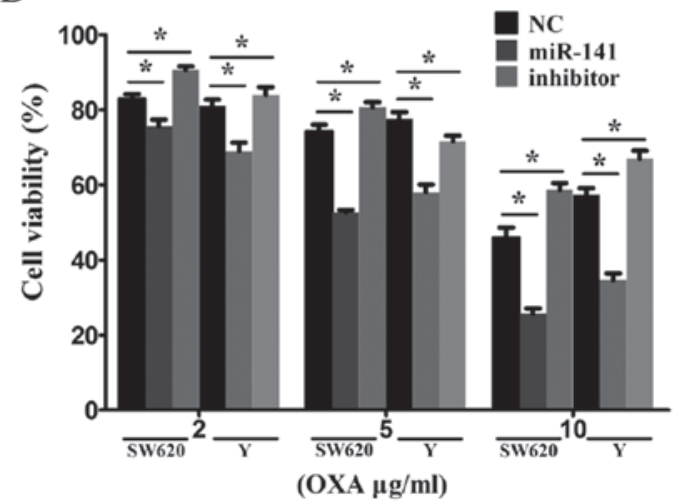

Figure 2. miR-141 exerts anti-tumor effects in CRC cells. Cells were transfected with a negative control mimic, an miR-141 mimic or an anti-miR-141 inhibitor (A) The cell proliferation rate and (B) IC50 values, were determined by measuring the absorbance at a wavelength of $490 \mathrm{~nm}$ following an MTS assay. Transfection with an miR-141 mimic enhanced the sensitivity of CRC cells to the chemotherapeutic agents (C) 5-FU and (D) OXA. "P<0.05. miR, microRNA; CRC, colorectal cancer; IC50, inhibitory concentration 50; 5-FU, 5-fluorouracil; OXA, oxaliplatin; NC, negative control.

anti-miR-141, Sox2, Oct4, Nanog and Bmi1 mRNA expression levels were elevated (Fig. 3A) indicating that miR-141 may promote stem cell differentiation.

Cyclin D2 is a novel target of miR-141 in CRC. Stem cell differentiation is associated with cell cycle progression. Flow cytometry revealed that, in SW620 and Y cells transfected with an miR-141 mimic, the proportion of cells in the G0/1 phase decreased, whereas the percentage in $\mathrm{S}$ phase increased (Fig. 3B).

Potential gene targets of miR-141 were analyzed using the TargetScan database (www.targetscan.org). Hundreds of potential miR-141 targets were further analyzed using the Kyoto Encyclopedia of Genes and Genomes pathway database (www.genome.jp/kegg/). Cyclin D2 was identified as a functional downstream target of miR-141. The cyclin D2 3' UTR contains highly conserved miR-141 binding sites (Fig. 4A). Western blotting revealed that cyclin D2 protein expression levels were decreased in cells following transfection with an miR-141 mimic, and increased following transfection with an anti-miR-141 inhibitor (Fig. 4B).

\section{Discussion}

miRNAs are currently undergoing extensive investigation due to their importance in the regulation of various developmental processes. They have been implicated in diverse diseases, including cancer. The present study investigated miRNAs that were differentially expressed in CSCs and differentiated cancer cells. Microarray analysis identified four miRNAs that were upregulated (miR-373, miR-1246-star, miR-494-star and miR-455-3p-star) and five that were downregulated (miR-29b, miR-638, miR-19a, miR-101 and miR-141) in CSCs compared with differentiated cancer cells. miR-373 has been revealed to be upregulated in breast cancer and to promote tumor invasion and metastasis (21). miR-1246-star promotes angiogenesis in CRC (22). miR-494-star has previously been demonstrated to be upregulated in CRC, and promotes cell migration and invasion by targeting the phosphatase and tensin homolog gene (23). miR-29b is downregulated in CRC and suppresses tumor growth and metastasis by inhibiting epithelial-mesenchymal transition (24); miR-638 is also downregulated and regulates the cell cycle by targeting tetraspanin 1 (25). miR-19a targets tissue factor to inhibit CRC migration and invasion (26). miR-101 exerts its antitumor effect via downregulating sphingosine kinase 1 (27). Low expression of miR-141 was identified in CSCs; however, its specific underlying mechanisms remained to be fully elucidated. In the present study, miR-141 inhibited the proliferation of CRC cells and the maintenance of stemness in CSC, enhancing drug susceptibility.

CSCs constitute a very small proportion of cancer cells that possess the capacity for self-renewal and differentiation. CSCs are hypothesized to be responsible for tumor recurrence, metastasis and chemotherapy resistance. CD133 is a well-established cell marker used to identify CSCs in colorectal cancer cell lines, including Y and HT29. These two cell lines were therefore selected to investigate the differential expression of miRNAs between CSCs and differentiated 

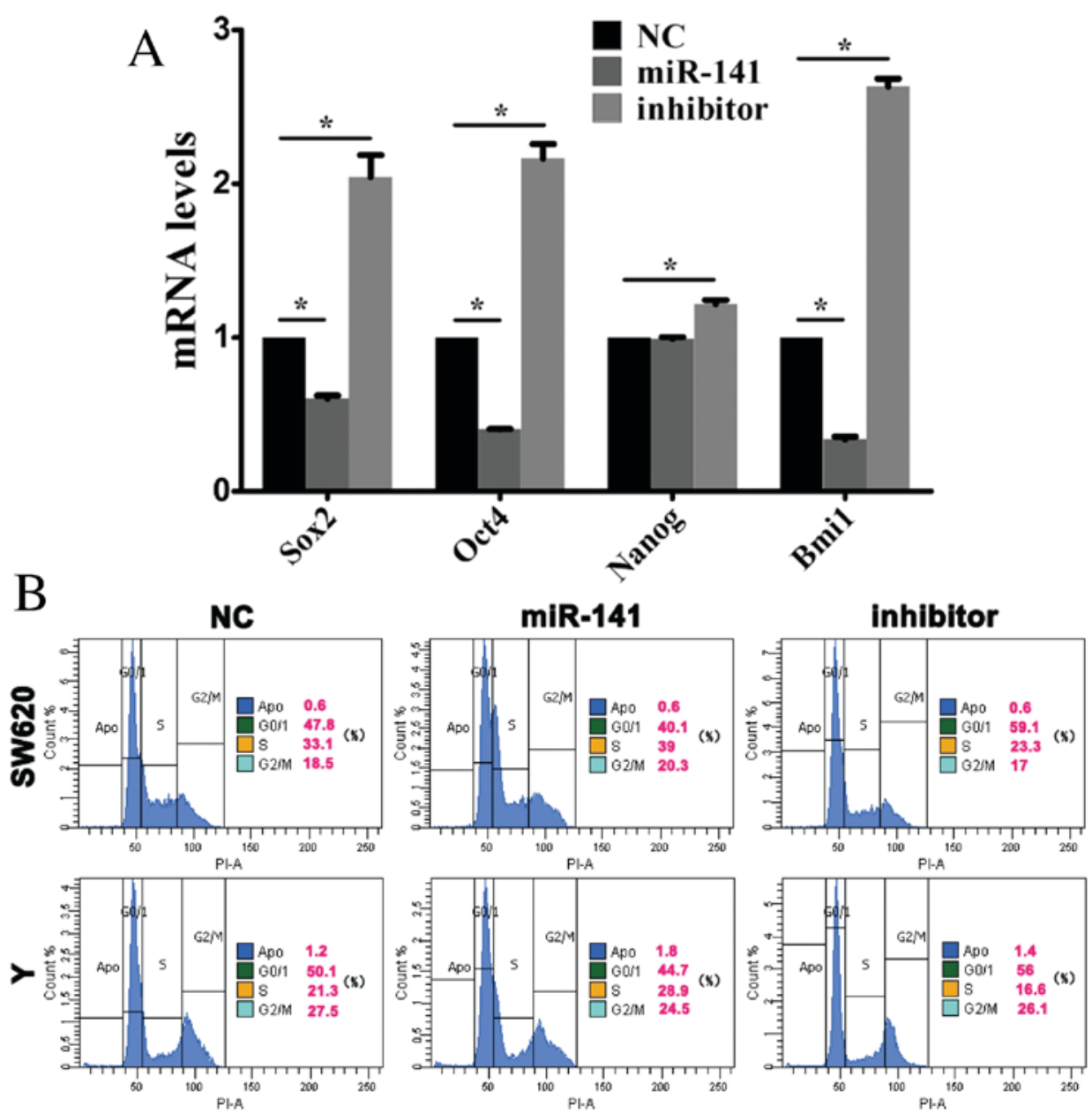

Figure 3. miR-141 inhibits the maintenance of cancer stem cell stemness. Cells were transfected with a negative control mimic, an miR-141 mimic or an anti-miR-141 inhibitor. (A) Sox2, Oct4, Nanog and Bmi1 mRNA expression levels in CRC cells were assessed by reverse transcription-quantitative polymerase chain reaction analysis. $\mathrm{P}<0.05$. (B) Cell cycle progression of CRC cells was assessed by flow cytometry. miR, microRNA; CRC, colorectal cancer; Sox 2 , sex determining region Y-box 2; Oct4, octamer-binding transcription factor 4; Nanog, nanog homeobox; Bmi1, B cell-specific Moloney murine leukemia virus integration site 1 ; NC, negative control.

A miRNA-141 3' GGUAGAAAUGGUCU-GUCACAAU 5' Cyclin D2 5'...GaggucuUaucuguUCAgUGUUA.... 3'

$\mathrm{B}$

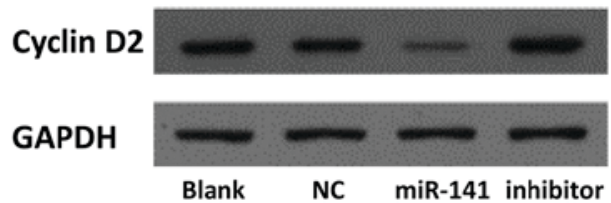

Figure 4. Cyclin D2 is a novel target of miR-141 in CRC. (A) The predicted binding site of miR-141 in the cyclin D2 3' untranslated region. (B) CRC cells were transfected with a negative control mimic, an miR-141 mimic or an anti-miR-141 inhibitor. The protein expression levels of cyclin D2 were detected by western blotting. GAPDH served as a loading control. CRC, colorectal cancer; miR, microRNA; NC, negative control.

cancer cells. miR-141 expression was decreased in CSCs, and this finding was confirmed by RT-qPCR. In addition, expression levels were decreased in CRC tissues compared with adjacent healthy tissues.

The function of miR-141 in CRC was subsequently investigated. miR-141 was demonstrated to inhibit cell proliferation and enhance chemotherapeutic drug sensitivity. Furthermore, cyclin D2 was identified as a target of miR-141 in CRC. Cyclin D2 is a member of the D-type cyclin protein family that regulates cell cycle progression. In mammalian cells, cyclin D forms a complex with cyclin-dependent kinase (CDK) 4 and CDK6 and regulates G1/S phase progression. Overexpression of cyclin D2 has been identified in various cancers and is associated with the proliferation of cancer cells (28). Increased expression of cyclin D2 has been detected in advanced CRC and was associated with reduced disease-free survival. In addition, cyclin D2 is regarded as an important regulator of self-renewal of human embryonic stem cells (hES) (29). Cyclin D2 is prominently expressed in pluripotent hES cells. The present study demonstrated that following inhibition of miR-141, cyclin D2 protein expression levels and the mRNA expression levels of stem cell-associated genes were elevated. These results indicated that miR-141 may affect the maintenance of stemness in CSC via cyclin D2.

Conventional cancer chemotherapy targets only differentiated cancer cells, which constitute the majority of the tumor. Although CSCs account for only a small fraction of total tumor cells, they remain quiescent in the G0 phase and are insensitive to chemotherapy (30). The present study revealed that miR-141 may promote CSC differentiation by reducing 
the expression of stem cell-associated genes, and thereby enhance their chemosensitivity.

In conclusion, the results of the present study demonstrated that miR-141 suppresses the growth of CRC cells via targeting cyclin D2, and inhibits the maintenance of CSC stemness, thereby enhancing drug susceptibility. Targeting miR-141 may therefore be a potential strategy for the treatment of CRC.

\section{Acknowledgements}

The present study was supported by the Zhejiang Provincial Natural Science Foundation of China (grant no. LY14H160027), and the Health and Family Planning Commission of Zhejiang Province (grant nos. 2015115856 and 2013KYA093).

\section{References}

1. Siegel RL, Miller KD and Jemal A: Cancer statistics, 2015. CA Cancer J Clin 65: 5-29, 2015

2. Todaro M, Alea MP, Di Stefano AB, Cammareri P, Vermeulen L, Iovino F, Tripodo C, Russo A, Gulotta G, Medema JP and Stassi G: Colon cancer stem cells dictate tumor growth and resist cell death by production of interleukin-4. Cell Stem Cell 1: 389-402, 2007.

3. Bartel DP: MicroRNAs: Genomics, biogenesis, mechanism, and function. Cell 116: 281-297, 2004.

4. Ambros V: The functions of animal microRNAs. Nature 431: 350-355, 2004

5. Lu J, Getz G, Miska EA, Alvarez-Saavedra E, Lamb J, Peck D, Sweet-Cordero A, Ebert BL, Mak RH, Ferrando AA, et al: MicroRNA expression profiles classify human cancers. Nature 435: 834-838, 2005.

6. Ma S, Tang KH, Chan YP, Lee TK, Kwan PS, Castilho A, Ng I, Man K, Wong N, To KF, et al: miR-130b Promotes CD133(+) liver tumor-initiating cell growth and self-renewal via tumor protein 53-induced nuclear protein 1. Cell Stem Cell 7: 694-707, 2010.

7. Visvader JE and Lindeman GJ: Cancer stem cells in solid tumours: Accumulating evidence and unresolved questions. Nat Rev Cancer 8: 755-768, 2008.

8. Dalerba P, Dylla SJ, Park IK, Liu R, Wang X, Cho RW, Hoey T, Gurney A, Huang EH, Simeone DM, et al: Phenotypic characterization of human colorectal cancer stem cells. Proc Natl Acad Sci USA 104: 10158-10163, 2007.

9. Yin S, Li J, Hu C, Chen X, Yao M, Yan M, Jiang G, Ge C, Xie H, Wan D, et al: CD133 positive hepatocellular carcinoma cells possess high capacity for tumorigenicity. Int J Cancer 120 1444-1450, 2007.

10. Wright $\mathrm{MH}$, Calcagno AM, Salcido CD, Carlson MD, Ambudkar SV and Varticovski L: Brcal breast tumors contain distinct CD44+/CD24- and CD133+ cells with cancer stem cell characteristics. Breast Cancer Res 10: R10, 2008.

11. Singh SK, Hawkins C, Clarke ID, Squire JA, Bayani J, Hide T, Henkelman RM, Cusimano MD and Dirks PB: Identification of human brain tumour initiating cells. Nature 432: 396-401, 2004

12. Ricci-Vitiani L, Lombardi DG, Pilozzi E, Biffoni M, Todaro M, Peschle C and De Maria R: Identification and expansion of human colon-cancer-initiating cells. Nature 445: 111-115, 2007.
13. Olempska M, Eisenach PA, Ammerpohl O, Ungefroren $\mathrm{H}$, Fandrich F and Kalthoff H: Detection of tumor stem cell markers in pancreatic carcinoma cell lines. Hepatobiliary Pancreat Dis Int 6: 92-97, 2007.

14. Collins AT, Berry PA, Hyde C, Stower MJ and Maitland NJ: Prospective identification of tumorigenic prostate cancer stem cells. Cancer Res 65: 10946-10951, 2005.

15. Bhatia M: AC133 expression in human stem cells. Leukemia 15 : 1685-1688, 2001

16. Wang YK, Zhu YL, Qiu FM, Zhang T, Chen ZG, Zheng S and Huang J: Activation of Akt and MAPK pathways enhances the tumorigenicity of CD133+ primary colon cancer cells. Carcinogenesis 31: 1376-1380, 2010.

17. Xu L, Li Q, Xu D, Wang Q, An Y, Du Q, Zhang J, Zhu Y and Miao Y: hsa-miR-141 downregulates TM4SF1 to inhibit pancreatic cancer cell invasion and migration. Int J Oncol 44: 459-466, 2014.

18. Grimholt RM, Urdal P, Klingenberg O and Piehler AP: Rapid and reliable detection of alpha-globin copy number variations by quantitative real-time PCR. BMC Hematol 14: 4, 2014.

19. Dalerba P, Cho RW and Clarke MF: Cancer stem cells: Models and concepts. Annu Rev Med 58: 267-284, 2007.

20. Wang Z, Oron E, Nelson B, Razis S and Ivanova N: Distinct lineage specification roles for NANOG, OCT4 and SOX2 in human embryonic stem cells. Cell Stem Cell 10: 440-454, 2012.

21. Huang Q, Gumireddy K, Schrier M, le Sage C, Nagel R, Nair S, Egan DA, Li A, Huang G, Klein-Szanto AJ, et al: The microRNAs miR-373 and miR-520c promote tumour invasion and metastasis. Nat Cell Biol 10: 202-210, 2008.

22. Yamada N, Tsujimura N, Kumazaki M, Shinohara H, Taniguchi K, Nakagawa Y, Naoe T and Akao Y: Colorectal cancer cell-derived microvesicles containing microRNA-1246 promote angiogenesis by activating Smad 1/5/8 signaling elicited by PML down-regulation in endothelial cells. Biochim Biophys Acta 1839: 1256-1272, 2014.

23. Sun HB, Chen X, Ji H, Wu T, Lu HW, Zhang Y, Li H and Li YM: miR-494 is an independent prognostic factor and promotes cell migration and invasion in colorectal cancer by directly targeting PTEN. Int J Oncol 45: 2486-2494, 2014.

24. Wang B, Li W, Liu H, Yang L, Liao Q, Cui S, Wang H and Zhao L: miR-29b suppresses tumor growth and metastasis in colorectal cancer via downregulating Tiam 1 expression and inhibiting epithelial-mesenchymal transition. Cell Death Dis 5: e1335, 2014.

25. Zhang J, Fei B, Wang Q, Song M, Yin Y, Zhang B, Ni S, Guo W, Bian Z, Quan C, et al: MicroRNA-638 inhibits cell proliferation, invasion and regulates cell cycle by targeting tetraspanin 1 in human colorectal carcinoma. Oncotarget 5: 12083-12096, 2014.

26. Yu G, Li H, Wang X, Wu T, Zhu J, Huang S, Wan Y and Tang J: MicroRNA-19a targets tissue factor to inhibit colon cancer cells migration and invasion. Mol Cell Biochem 380: 239-247, 2013.

27. Chen MB, Yang L, Lu PH, Fu XL, Zhang Y, Zhu YQ and Tian Y: MicroRNA-101 down-regulates sphingosine kinase 1 in colorectal cancer cells. Biochem Biophys Res Commun 463: 954-960, 2015.

28. Meyyappan M, Wong H, Hull C and Riabowol KT: Increased expression of cyclin D2 during multiple states of growth arrest in primary and established cells. Mol Cell Biol 18: 3163-3172, 1998.

29. Becker KA, Ghule PN, Lian JB, Stein JL, van Wijnen AJ and Stein GS: Cyclin D2 and the CDK substrate p220 (NPAT) are required for self-renewal of human embryonic stem cells. J Cell Physiol 222: 456-464, 2010.

30. Wang J, Yang M, Li Y and Han B: The role of MicroRNAs in the chemoresistance of breast cancer. Drug Dev Res 76: 368-374, 2015. 